\title{
SAND99.0123J
}

\section{Charge Accumulation at a Threading Edge Dislocation in GaN}

\author{
K. Leung, A. F. Wright, and E. B. Stechel ${ }^{1}$ \\ Sandia National Laboratories, MS 1421, Albuquerque, N.M. 87185 \\ ${ }^{1}$ Ford Motor Company, MD 3028/SRL, Dearborn, MI 48121-2053
}

(December 19, 1998)

\begin{abstract}
We have performed Monte Carlo calculations to determine the charge accumulation on threading edge dislocations in GaN as a function of the dislocation density and background dopant density. Four possible core structures have been examined, each of which produces defect levels in the gap and may therefore act as electron or hole traps. Our results indicate that charge accumulation, and the resulting electrostatic interactions, can change the relative stabilities of the different core structures. Structures having $\mathrm{Ga}$ and $\mathrm{N}$ vacancies at the dislocation core are predicted to be stable under nitrogen-rich and gallium-rich growth conditions, respectively. Due to dopant depletion at high dislocation density and the multitude of charge states, the line charge exhibits complex crossover behavior as the dopant and dislocation densities vary.
\end{abstract}

Gallium nitride films grown on sapphire substrates typically contain between $10^{8}$ and $10^{10}$ threading dislocations per $\mathrm{cm}^{2}$ as a result of the substantial film-substrate chemical and lattice mismatch. ${ }^{1}$ Nevertheless, it has been possible to fabricate bright and efficient light-emitting diodes from films composed of GaN alloyed with $\mathrm{InN}$ and AlN. ${ }^{2}$ This success led several researchers to speculate early on that threading dislocations in GaN might not act as efficient minority-carrier recombination sites. ${ }^{1}$ Recent experimental studies, however, have confirmed that there is significant optical ${ }^{3}$ and electrical ${ }^{4}$ activity associated with these defects. In particular, results from a recent scanning-capacitance microscopy study ${ }^{5}$ suggest that dislocations are negatively charged in $n$-type GaN, and studies of transverse mobility in $n$-type $\mathrm{GaN}_{\text {films }} \mathrm{s}^{6,7}$ indicate that electrons are scattered from these negatively charged dislocations.

Recent charge-state calculations for $\mathrm{AlN}$ and $\mathrm{GaN}^{8}$ indicate that threading edge dislocations produce defect levels in the forbidden energy gap. These calculations provide estimates for these defect levels. In agreement with experimental studies, edge dislocations are predicted to be negatively charged in $n$-type GaN. However, the amount of charge accumulation could not be quantified because electrostatic interactions between charged defect sites and between defect sites and ionized dopants were not included in the calculations. In this study, we treat coulomb interactions explicitly and therefore are able to predict the amount of charge accumulation on an edge dislocation under a variety of doping conditions.

We examine the zero-temperature behavior of threading dislocations using simulated annealing. ${ }^{9}$ The simulation cell contains one dislocation and is of lateral dimension $\sigma_{\mathrm{dis}}^{-1 / 2}$, where $\sigma_{\mathrm{dis}}$ is the dislocation density. Electrons, modelled as point charges, can transfer between dopants (donors or acceptors) and defect levels at the dislocation cores, and among the dopants themselves. The dislocation consists of 50-1000 defect sites situated $5.185 \AA$ apart. We have examined the four dislocation core structures previously considered in first prin- ciples studies: full core, open core, Ga-vacancy, and Nvacancy. ${ }^{8}$ The possible charge states in each core structure are bracketed by the minimum and maximum values deduced from first principles calculations. The energy levels of these charge states, neglecting coulomb interactions, are listed in Table I. The coulomb interactions among charged defects and ionized dopants are screened using an empirical, bulk, orientationally averaged static dielectric constant of $\epsilon_{o}=10$. Each dopant is randomly placed within a preassigned volume equal to the inverse of the dopant density $\rho_{d}$. The ionization energy (electron affinity) of electron donors (acceptors) are taken from experimental values; see Table I. We apply periodic boundary conditions in all three spatial dimensions and treat the image charges using the Ewald sum technique. We find that the effect of dopant positions on simulation results is negligible. By considering more than one dislocation per simulation cell, we also find that correlation between dislocations is negligible. We expect the effect of finite temperature will not be important because defect levels are widely separated in the band gap.

The existence of multiple charge states suggests that charge modulation along the dislocation line might be energetically favorable. For example, in the isolated $\mathrm{Ga}$ vacancy structure, if the charges were discrete, alternating $-3 /+3$ charges are favored over the $0 / 0$ charge configuration by $\sim 0.24 \mathrm{eV}$ per site. However, first principles studies of core structure cells doubled along the dislocation direction indicate that the excess charges are always delocalized, due to (1) large dispersions in the low-lying impurity bands; and (2) unfavorable strain field interactions associated with unlike charge states on adjacent core structures. Based on the above, we treat the charges residing in defect levels as delocalized and uniformly distributed over the length of the dislocation, and we model these charges as a continuous variable $\lambda$.

In the limit of small line charge $\lambda$, our work is similar to the model derived by Read ${ }^{10}$ and widely used for making line charge estimates. ${ }^{6}$ Our model improves upon that analytical work in that it can treat (1) multiple charge 


\section{DISCLAIMER}

This report was prepared as an account of work sponsored by an agency of the United States Government. Neither the United States Government nor any agency thereof, nor any of their employees, make any warranty, express or implied, or assumes any legal liability or responsibility for the accuracy, completeness, or usefulness of any information, apparatus, product, or process disclosed, or represents that its use would not infringe privately owned rights. Reference herein to any specific commercial product, process, or service by trade name, trademark, manufacturer, or otherwise does not necessarily constitute or imply its endorsement, recommendation, or favoring by the United States Government or any agency thereof. The views and opinions of authors expressed herein do not necessarily state or reflect those of the United States Government or any agency thereof. 


\section{DISCLAIMER}

Portions of this document may be illegible in electronic image products. Images are produced from the best available original document. 
states; (2) explicit interactions between dislocations; and, in principle, (3) the entropy of electron hopping among dopants at high temperature.
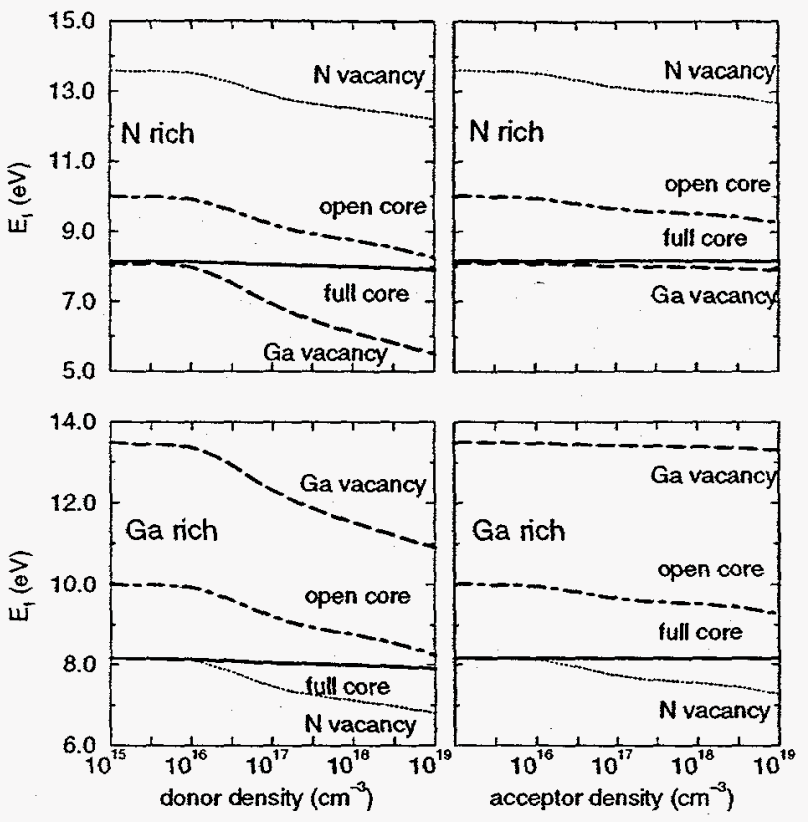

FIG. 1. Formation energies for core structures vs. dopant density. Left-hand side panels: $n$-type; right-hand side panels: p-type. Solid line - full core; dotted - N-vacancy; dashed - Ga-vacancy; dot-dashed - open core. $\sigma_{\text {dis }}=10^{10} \mathrm{~cm}^{-2}$ in these plots.

The predicted formation energies $\left(E^{f}\right)$ per $c$ lattice constant in $n$-type materials are shown in the left panels of Fig. 1 for N-rich and Ga-rich growth conditions. $E^{\mathrm{f}}$ incorporates the defect and donor energy levels (Table I), electrostatic contributions, and chemical potentials for the reference $\mathrm{Ga}$ metal, $\mathrm{N}_{2}$ gas, and bulk $\mathrm{GaN}^{8}$ Note that, due to the long ranged nature of the strain fields, the relative formation energies are relevant while the absolute values are not. We find that the Ga-vacancy structure is favorable under $\mathrm{N}$-rich growth conditions and the $\mathrm{N}$-vacancy structure is favored under Ga-rich growth conditions over almost the entire range of attainable donor densities. $E^{\mathrm{f}}$ decreases with increasing $\rho_{d}$ because of a combination of (1) the energy gain in transferring more electrons from donors to defects; and (2) a better screening of the coulomb repulsion between defect sites at large $\rho_{d}$. The full core structure formation energy is almost. independent of $\rho_{d}$ because the available defect levels are high in energy, and only small net charges accumulate on the defect sites.

The $\rho_{d}$ dependence of $E^{\mathrm{f}}$ falls into two regimes. For small $\rho_{d}$, such that the number of defect sites far exceeds the number of donors, all dopants are ionized and depleted by charge transfer to the dislocation defect sites (Fig. 2). In this limit, $\lambda \rightarrow 0$, the Fermi energy is pinned close to the partially occupied defect level, and the or- dering of $E^{\mathrm{f}}$ for different core structures closely resembles that found in Ref. 8, where defect-defect interactions are omitted.

At large $\rho_{d}$, there is an abundance of free charges. As these charges accumulate on the dislocation, they build up an electrostatic repulsion that pushes the Fermi energy upwards, away from the isolated defect. levels until it coincides with the donor level. The defect levels can be thought of as strongly renormalized by the coulomb repulsion. In this regime, the coulomb interaction between dislocation lines is effectively screened out by the ionized dopants. $\lambda$ becomes independent of $\sigma_{\text {dis }}$, and the dislocations behave as though they are at infinite dilution at the appropriate $\rho_{d}$ (Fig. 2). $E^{\mathrm{f}}$ is strongly modified by the coulomb interactions in this regime.
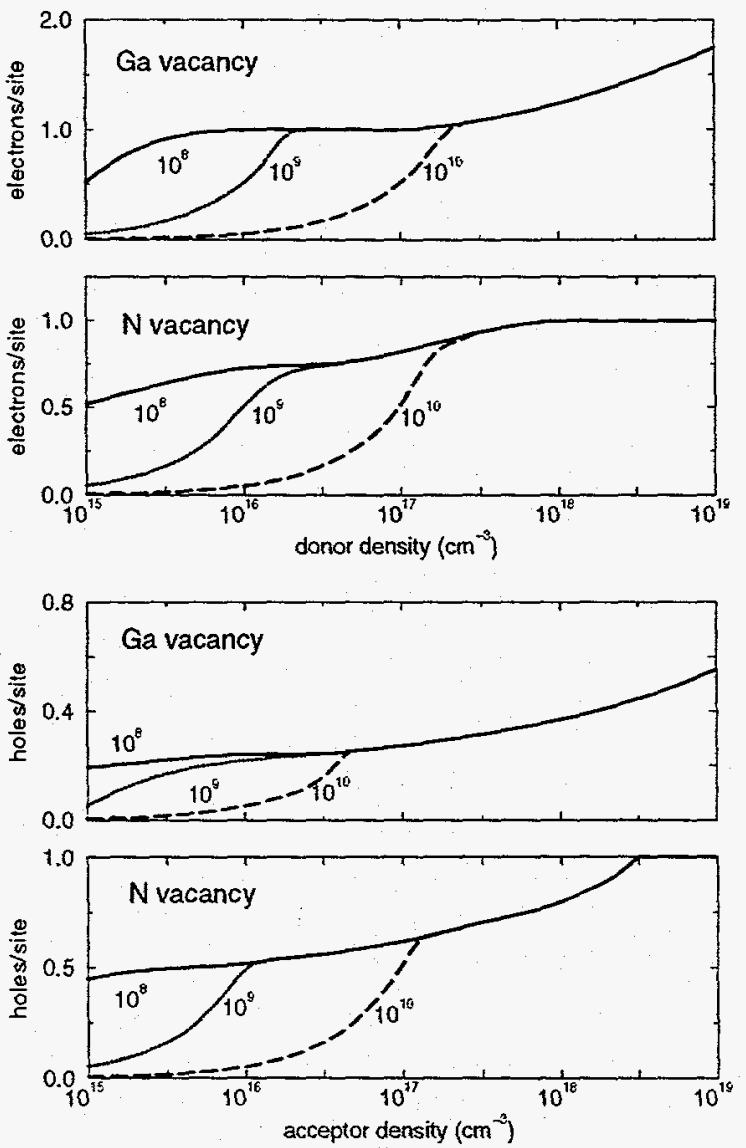

FIG. 2. Line charges per site for the energetically favorable Ga- and N-vacancy structures as functions of dopant density. Upper two panels: $n$-type; lower two panels: $p$-type. Solid line $-\sigma_{\text {dis }}=10^{8}\left(\mathrm{~cm}^{-2}\right)$; dotted $-10^{9}$; dashed $-10^{10}$.

The change in slope of $E^{\mathrm{f}}$ and the $\lambda$-upturn in Fig. 2 at $\rho_{d} \sim 10^{16} \mathrm{~cm}^{-3}$ and $\sigma_{\text {dis }}=10^{10} \mathrm{~cm}^{-2}$ reflect the crossover between the two regimes. In this crossover region, the Fermi energy is intermediate between the defect and donor levels. Significantly, $\rho_{d} \sim 10^{16} \mathrm{~cm}^{-3}$ is also the unintentional doping density obtained using current growth techniques. Note that the donor-depletion crossover in general does not coincide with the complete 
filling of a level. It moves to larger $\rho_{d}$ as $\sigma_{\text {dis }}$ increases.

The Ga- and N-vacancy structures (favorable under $\mathrm{N}$ - and Ga-rich growth conditions respectively) exhibit plateaus at $\lambda=1$ when the -1 charge states are fully occupied. The Fermi energies reside above the donor level and gaps develop in the defect densities of states, until the next higher defect level becomes occupied. The predicted line charge for the Ga-vacancy structure is in qualitative accord with $\lambda$ inferred from scattering experiments. ${ }^{7}$ Unlike Ref. 6 , which applies the model of Read ${ }^{10}$ and predicts a discontinuous slope near $\lambda=1$ for $n$-type materials, a smooth approach to charge saturation is observed in our results.

Note that $E^{f}$ in Fig. 1 are computed for $\sigma_{\text {dis }}=10^{10}$ $\mathrm{cm}^{-2}$. At lower $\sigma_{\text {dis }}$, there are more free charges available, the threshold for depletion is pushed to lower $\rho_{d}$, and the charging induced separation between $E^{f}$ for various core structures widens. In other words, the $\mathrm{Ga}^{-}$and $\mathrm{N}$-vacancy structures become even more energetically favorable at low $\sigma_{\text {dis }}$.

The right panels in Fig. 1 depict the formation energies in p-type material. All core structures, except the full core, can act as electron donors. The $E^{\mathrm{f}}$ trends are similar to those in $n$-type materials. At low acceptor density, all dopants (acceptors) gain an electron from the dislocation defect sites, and the Fermi energy is pinned close to the partially filled defect level. The $E^{\mathrm{f}}$ ordering follows that in uncharged threading dislocations ${ }^{8}$ at that Fermi energy, At high acceptor density, the positive charge built up on the dislocation lowers the electrostatic potential of electrons there, and the Fermi energy falls to the acceptor chemical potential. The Ga vacancy structure is predicted to be more favorable than the full core by a small fraction of an electron volt in $p$-type material. That magnitude is probably within the error margin of the present model. Experimentally, the full core and $\mathrm{Ga}$ vacancy structures can be distinguished by their different line charges. (The full core structure is uncharged.) The crossover in $E^{\mathrm{f}}$ is more gradual for $p$-type than $n$-type material, because the isolated defect levels are generally closer to the acceptor level than the shallow donor levels (Table I), yielding a smaller gain in charge transfer. As $\sigma_{\text {dis }}$ decreases, the Ga vacancy structure becomes progressively more favorable than the full core.

In conclusion, the predictions of our model can be guides to the characterization of edge dislocation core structures. In particular, we predict that $\mathrm{N}$-rich growth conditions favor the Ga-vacancy structure, while Ga-rich growth conditions favor the $\mathrm{N}$-vacancy structure. The predicted line charge can be used to analyze the the Hall mobility in the charged dislocation-dominated scattering regime. ${ }^{6}$ Coulomb effects on the Fermi energy described in this work can also have significant implications for optical transitions involving dislocation defect levels.

We would like to thank Prof. D.C. Look for sharing his data prior to publication. The first principles density functional calculations are performed using the Vi- enna molecular simulation package. ${ }^{11}$ This work was supported by the U.S. Department of Energy under Contract DE-AC04-94AL85000. Sandia is a multiprogram laboratory operated by Sandia Corporation, a Lockheed Martin Company, for the U.S. Department of Energy.

${ }^{1}$ S. D. Lester, F. A. Ponce, M. G. Craford, and D. A. Steigerwald, Appl. Phys. Lett. 66, 1249 (1995).

${ }^{2}$ S. Nakamura, T. Mukai, and M. Senoh, Appl. Phys. Lett. 64, 1687 (1993); S. Nakamura, M. Senoh, N. Iwasa, and S. Nagahama, Appl. Phys. Lett. 67, 1868 (1995); S. Nakamura, M. Senoh, N. Iwasa, S. Nagahama, T. Yamada, and T. Mukai, Jpn. J. Appl. Phys. 34, L1332 (1995).

${ }^{3}$ S. J. Rosner, E. C. Carr, M. J. Ludowise, G. Girolami, and H. I. Erikson, Appl. Phys. Lett. 70, 420 (1997); T. Sugahara, H. Sao, M. Hao, Y. Naoi, S. Kurai, S. Tottori, K. Yamashita, K. Nishino, L. T. Romano, and S. Sakai, Jpn. J. Appl. Phys. 37, L398 (1998).

${ }^{4}$ C. Youtsey, L. T. Romano, and I. Adesida, Appl. Phys. Lett. 73, 797 (1998).

E P. J. Hansen, Y. E. Strausser, A. N. Erickson, E. J. Tarsa, P. Kozodoy, E. G. Brazel, J. P. Ibbetson, U. Mishra, V. Narayanamurti, S. P. DenBaars, and J. S. Speck, Appl. Phys. Lett. 72, 2247 (1998).

${ }^{6}$ N. G. Weinmann, L. F. Eastman, D. Doppalapudi, H. M. Ng, and T. D. Moustakas, J. Appl. Phys. 83, 3656 (1998); H. M. Ng, D. Doppalapui, T. D. Moustakas, N. G. Weinmann, and L. F. Eastman, Appl. Phys. Lett. 73, 821 (1998).

${ }^{7}$ D. C. Look and J. R. Sizelove (submitted for publication).

${ }^{8}$ A. F. Wright and J. Furthmüller, Appl. Phys. Lett. 72, 3467 (1998); A. F. Wright and U. Grossner, Appl. Phys. Lett. 73, 2751 (1998). Note that the $\mathrm{N}_{2}$ chemical potential used herein is shifted from the value in these publications by the difference between LDA and experimental chemical potentials.

${ }^{9}$ Applied Simulated Annealing, edited by R. V. V. Vidal (Springer-Verlag, Berlin, 1993).

${ }^{10}$ W. T. Read, Philo. Mag. 45, 775 (1954).

${ }^{11}$ G. Kresse and J. Hafner, Phys. Rev. B 47, 558 (1993); 49, 14251 (1994); G. Kress and J. Furthmüller, Phys. Rev. B 54, 11169 (1996).

\begin{tabular}{||c|c|c|c|c|c|c|c|c||}
\hline \hline charge state & +3 & +2 & +1 & 0 & -1 & -2 & -3 & -4 \\
\hline Full core & & & & 0.00 & 2.78 & 2.57 & & \\
open core & & 0.00 & 0.32 & 0.83 & 1.39 & 1.55 & 2.89 & 3.45 \\
Ga vacancy & 0.00 & 0.32 & 0.35 & 0.44 & 0.72 & 1.16 & 1.74 & \\
N vacancy & & & 0.00 & 1.87 & 1.56 & 3.15 & 3.45 & \\
\hline
\end{tabular}

TABLE I. Defect levels in $\mathrm{eV}$, referenced to the valence band maximum. The donor ( $\mathrm{Si}$ ) and acceptor $(\mathrm{Mg})$ dopant levels are at 3.45 and $0.16 \mathrm{eV}$, respectively. For details, see Ref. 8 . 